\title{
Westbrook's Molecular Gun: Discovery of Near-Infrared Microstructures and Molecular Bullets in AFGL 618
}

\author{
Toshiya Ueta, David Fong, \& Margaret Meixner \\ Department of Astronomy, MC-221, \\ University of Illinois at Urbana-Champaign, Urbana, IL 61801, USA \\ ueta@astro.uiuc.edu,d-fong@astro.uiuc.edu, meixner@astro.uiuc.edu
}

We present high-sensitivity near-IR images of a carbon-rich proto-planetary nebula, AFGL 618, obtained with the Infrared Camera and Spectrograph (IRCS) mounted on the $8.2 \mathrm{~m}$ Subaru Telescope. The deep near-IR images have revealed "bullets" and "horns" extending farther out from the edges of the previously known bipolar nebulosities that consist of dust-scattered star light component and shock-excited line emission component. That these bullets and horns represent the positions from which [Fe II] IR lines arise is strongly suggested from the spatial coincidence between these near-IR microstructures and the optical collimated outflow structure observed by the recent HST/WFPC2 imaging, together with the previous detection of shock-excited, forbidden IR lines of atomic species at those locations. At these positions of the near-IR mincrostructures, we have also discovered CO clumps moving at $>200 \mathrm{~km} \mathrm{~s}^{-1}$ from our re-analysis of the existing ${ }^{12} \mathrm{CO} J=1-0$ data obtained with the Berkeley-Illinois-Maryland Association (BIMA) interferometer array. These findings indicate that fast-moving $\mathrm{CO}$ clumps seem to be impinging upon the surrounding ambient circumstellar shell, thereby causing shocked emission regions which manifest themselves as the near-IR microstructures at the shock interface. To deepen our understanding of the connection between the near-IR microstructures and the $\mathrm{CO}$ outflow structure, we are currently conducting higher resolution observation in CO lines with the BIMA array. 